\title{
Determinantal Representation of the Time-Dependent Stationary Correlation Function for the Totally Asymmetric Simple Exclusion Model ${ }^{\star}$
}

\author{
Nikolay M. BOGOLIUBOV \\ St. Petersburg Department of V.A. Steklov Mathematical Institute, \\ Fontanka 27, St. Petersburg 191023, Russia \\ E-mail: bogoliub@pdmi.ras.ru \\ Received October 30, 2008, in final form April 14, 2009; Published online April 23, 2009 \\ doi:10.3842/SIGMA.2009.052
}

\begin{abstract}
The basic model of the non-equilibrium low dimensional physics the so-called totally asymmetric exclusion process is related to the 'crystalline limit' $(q \rightarrow \infty)$ of the $S U_{q}(2)$ quantum algebra. Using the quantum inverse scattering method we obtain the exact expression for the time-dependent stationary correlation function of the totally asymmetric simple exclusion process on a one dimensional lattice with the periodic boundary conditions.
\end{abstract}

Key words: quantum inverse method; algebraic Bethe ansatz; asymmetric exclusion process

2000 Mathematics Subject Classification: 82C23; 81R50

\section{Introduction}

The study of quantum exactly solvable models within the framework of the quantum inverse scattering method (QISM) [1, 2, 3] has led to an algebraic structures which are $q$-deformations of the universal enveloping algebras $[4,5]$. These deformed structures are usually called now 'quantum groups' or 'quantum algebras'. Integrable models associated with the 'crystalline limit' $(q \rightarrow \infty)$ [6] of these algebras play an important role in enumerative combinatorics $[7,8]$ and non-equilibrium low dimensional physics $[9,10]$.

The algebraic approach to the calculation of the correlation functions for the integrable models within the frames of the (QISM) was developed in the late eighties of the last century [11] advantage of this approach is that the form of the final answers depends on the $R$-matrix of the model and not on the particular realization of the operators intertwained by this matrix. It is also important that the operator averages are calculated over the eigenstates of the generating function of the integrals of motion.

The correlation functions for the boson model associated with the 'crystalline limit' of the $X X Z R$-matrix were calculated in $[12,13]$ where it was shown that they have the determinantal form.

The totally asymmetric simple exclusion process (TASEP) is an integrable model that is connected with the 'crystalline limit' of the $X X Z R$-matrix. It is one of the most studied models of the low dimensional non-equilibrium physics. The Bethe ansatz approach to the solution of the model was developed in the papers $[14,15,16,17,18,19]$. The relation of the model to the QISM has been studied in [20,21, 22], the model with the twisted boundary conditions within the frames of this method was considered in [23], and the asymmetric exclusion process in [24]. The dynamical properties of TASEP are intensively studied (see, e.g., [25, 26, 27] and

${ }^{\star}$ This paper is a contribution to the Proceedings of the XVIIth International Colloquium on Integrable Systems and Quantum Symmetries (June 19-22, 2008, Prague, Czech Republic). The full collection is available at http://www.emis.de/journals/SIGMA/ISQS2008.html 
the references in these papers). The KPZ scaling limit of TASEP was considered, in particular, in $[14,28,29]$.

In the present paper we shall consider the TASEP on a periodic lattice with the finite number of sites and with the finite number of particles. We shall represent the form-factors of the local operators in the determinantal form using the algebraic approach of the QISM. This result may be considered as the main result of the present paper.

The paper is structured as follows. In Section 2, the definition of the model is given. The algebraic approach to the solution of the model is presented in Section 3, in this section the stationary states are expressed through the state vectors of the model and the scalar product of the state vectors is expressed in the determinantal form. In Section 4, the calculation of the averages of the local projection operators over the state vectors of the model defined on a lattice with $M$ sites is reduced to the calculation of the scalar product of the state vectors of the model defined on the $M-1$ sites. In Section 5, the well known results on the Bethe ansatz solution are presented. The answer, based on the results of the section 6 , for the time-dependent stationary correlation function is obtained. Finally, conclusive remarks are given in Section 7.

\section{Totally asymmetric simple exclusion model}

The totally asymmetric exclusion process describes a system of $N$ particles on a periodic ring with $M$ sites labelled $i=M, M-1, \ldots, 2,1$. The particles move randomly only in one direction. We shall choose this direction from right to left. The exclusion rule forbids to have more than one particle per site.

This process can be conveniently represented using the spin description - in each lattice site the spin-up state corresponds to the empty site $|0\rangle$, the spin-down state corresponds to the occupied one $|1\rangle$. The configuration of $N$ particles $(2 N \leq M)$ located on the sites $M \geq m_{1}>$ $m_{2}>\cdots>m_{N} \geq 1$ is given by the basis vector

$$
\left|m_{1}, m_{2}, \ldots, m_{N}\right\rangle=\sigma_{m_{1}}^{-} \sigma_{m_{2}}^{-} \cdots \sigma_{m_{N}}^{-}|\Omega\rangle,
$$

where the generating state $|\Omega\rangle$ is the state with all spins up

$$
|\Omega\rangle=\otimes_{i=1}^{M}|0\rangle_{i}
$$

The described process is defined by a non-Hermitian Hamiltonian [14]:

$$
H=-\sum_{j=1}^{M}\left\{\sigma_{j+1}^{-} \sigma_{j}^{+}+\frac{1}{4}\left(\sigma_{j+1}^{z} \sigma_{j}^{z}-1\right)\right\} .
$$

Here $\sigma^{ \pm, z}$ are the Pauli matrices, the matrix with subindex $j$ acts nontrivially only in the $j$-th spin space of the total space of states of the chain $\left(\mathbb{C}^{2}\right)^{\otimes M}: s_{j}=I \otimes \cdots \otimes I \otimes s \otimes I \otimes \cdots \otimes I$, and the periodic boundary conditions are assumed: $\sigma_{n}=\sigma_{n+M}$.

In the matrix representation $-H$ is the Markov matrix that controls the time evolution of the probability measure.

The first term of the Hamiltonian describes the hoppings of particles

$$
\sigma_{j+1}^{-} \sigma_{j}^{+}|0\rangle_{j+1}|1\rangle_{j}=|1\rangle_{j+1}|0\rangle_{j}
$$

The second term counts the number of the allowed jumps of the particles with hard-core repulsion.

In our paper we shall consider the time-dependent stationary correlation function [14]

$$
Z_{N}^{-1}\left\langle S_{N}\left|s_{1} e^{-|t| H} s_{m}\right| S_{N}\right\rangle,
$$


where the projection operator

$$
s_{k}=\frac{1}{2}\left(1+\sigma_{k}^{z}\right)
$$

has value 1 if there is no particle at site $k$ and 0 otherwise. In the steady state $\left|S_{N}\right\rangle$ every spin configuration with $N$ spins down has the equal weight:

$$
\left|S_{N}\right\rangle=\sum_{M \geq m_{1}>m_{2}>\cdots>m_{N} \geq 1}\left|m_{1}, \ldots, m_{N}\right\rangle
$$

This state is a ground state of Hamiltonian with the eigenvalue zero:

$$
H\left|S_{N}\right\rangle=0
$$

The left eigenvector of $H$ with the eigenvalue zero is

$$
\left\langle S_{N}\right|=\sum_{M \geq m_{1}>m_{2}>\cdots>m_{N} \geq 1}\left\langle m_{1}, \ldots, m_{N}\right| .
$$

The total number of configurations in the ground state

$$
Z_{N}=\left\langle S_{N} \mid S_{N}\right\rangle=\frac{M !}{N !(M-N) !}
$$

One can express the right steady state in the following form [30]:

$$
\left|S_{N}\right\rangle=\mathcal{P}_{R}^{N}|\Omega\rangle
$$

where $\mathcal{P}_{R}^{N}$ is the $N$-th power of operator

$$
\mathcal{P}_{R}=\sum_{k=1}^{M} s_{M} \cdots s_{k+1} \sigma_{k}^{-},
$$

$s_{j}$ are projection operators (3) and $\sigma_{k}^{-}$are Pauli matrices. Similarly for the left steady state one has

$$
\left\langle S_{N}\right|=\langle\Omega| \mathcal{P}_{L}^{N}
$$

with the operator $\mathcal{P}_{L}$ equal to

$$
\mathcal{P}_{L}=\sum_{k=1}^{M} \sigma_{k}^{+} s_{k-1} \cdots s_{1} .
$$

\section{Solution of the model}

To the solution of the model we shall apply the quantum inverse scattering method $[1,2,3]$. The $L$-operator of the considered model $[21,23]$ is a $2 \times 2$ matrix with the entries acting on the space of states of an $M$-site spin- $\frac{1}{2}$ chain:

$$
L(n \mid u)=\left(\begin{array}{cc}
u s_{n} & \sigma_{n}^{-} \\
\sigma_{n}^{+} & u I-u^{-1} s_{n}
\end{array}\right)=s s_{n}+(I-s)\left(u I-u^{-1} s_{n}\right)+\sigma^{-} \sigma_{n}^{+}+\sigma^{+} \sigma_{n}^{-},
$$

where the parameter $u \in \mathbb{C}$. 
The $L$-operator (6) satisfies the intertwining relation

$$
R(u, v)(L(n \mid u) \otimes L(n \mid v))=(L(n \mid v) \otimes L(n \mid u)) R(u, v),
$$

in which $R(u, v)$ is the $4 \times 4$ matrix

$$
R(u, v)=\left(\begin{array}{cccc}
f(v, u) & 0 & 0 & 0 \\
0 & g(v, u) & 1 & 0 \\
0 & 0 & g(v, u) & 0 \\
0 & 0 & 0 & f(v, u)
\end{array}\right)
$$

with

$$
f(v, u)=\frac{u^{2}}{u^{2}-v^{2}}, \quad g(v, u)=\frac{u v}{u^{2}-v^{2}} .
$$

In the quantum group theory this $R$-matrix is known as the 'crystal base' $R$-matrix [6].

The monodromy matrix is the product of $L$-operators

$$
T(u)=L(M \mid u) L(M-1 \mid u) \cdots L(1 \mid u)=\left(\begin{array}{cc}
A(u) & B(u) \\
C(u) & D(u)
\end{array}\right) .
$$

The commutation relations of the matrix elements of the monodromy matrix are given by the same $R$-matrix (7)

$$
R(u, v)(T(u) \otimes T(v))=(T(v) \otimes T(u)) R(u, v) .
$$

In the explicit form the most important of these relations for us are

$$
\begin{aligned}
& C(u) B(v)=g(u, v)\{A(u) D(v)-A(v) D(u)\}, \\
& A(u) B(v)=f(u, v) B(v) A(u)+g(v, u) B(u) A(v), \\
& D(u) B(v)=f(v, u) B(v) D(u)+g(u, v) B(u) D(v), \\
& {[B(u), B(v)]=[C(u), C(v)]=0 .}
\end{aligned}
$$

The transfer matrix $\tau(u)$ is the matrix trace of the monodromy matrix

$$
\tau(u)=u^{-M} \operatorname{tr} T(u)=u^{-M}(A(u)+D(u)) .
$$

The relation (9) means that $[\tau(u), \tau(v)]=0$ for arbitrary values of parameters $u, v$.

The cyclic shift operator $\tau$ in the total spin space $\left(\mathbb{C}^{2}\right)^{\otimes M}$ is expressed through the transfer matrix:

$$
\tau \equiv \tau(1)=\Pi_{12} \Pi_{23} \cdots \Pi_{M-1 M}
$$

Here

$$
\Pi_{m n}=s_{m} s_{n}+\left(I-s_{m}\right)\left(I-s_{n}\right)+\sigma_{m}^{-} \sigma_{n}^{+}+\sigma_{m}^{+} \sigma_{n}^{-},
$$

is the permutation operator: $\Pi_{m n} \sigma_{m}=\sigma_{n} \Pi_{m n}$. The shift operator shifts the site indices

$$
\tau^{n-1} \sigma_{1} \tau^{1-n}=\sigma_{n}
$$

and possesses the property $\tau^{M}=I$.

In terms of the transfer matrix (11) the Hamiltonian (1) is given by

$$
H=-\left.\frac{1}{2} \tau^{-1}(1) \frac{\partial}{\partial u} \tau(u)\right|_{u=1} .
$$


The right state vector of the model is the vector generated by the multiple action of operators $\tilde{B}(u) \equiv u^{-(M-1)} B(u)$ on the generating state $|\Omega\rangle=\otimes_{i=1}^{M}|0\rangle_{i}$

$$
\left|\Psi\left(u_{1}, u_{2}, \ldots, u_{N}\right)\right\rangle=\prod_{i=1}^{N} \tilde{B}\left(u_{i}\right)|\Omega\rangle .
$$

The generating state is annihilated by the operator $C(u)$

$$
C(u)|\Omega\rangle=0,
$$

and it is an eigenvector of operators $A(u)$ and $D(u)$,

$$
A(u)|\Omega\rangle=\alpha(u)|\Omega\rangle, \quad D(u)|\Omega\rangle=\delta(u)|\Omega\rangle
$$

with the eigenvalues

$$
\alpha(u)=u^{M}, \quad \delta(u)=\left(u-u^{-1}\right)^{M} .
$$

The left state vector is equal to

$$
\left\langle\Psi\left(u_{1}, u_{2}, \ldots, u_{N}\right)\right|=\langle\Omega| \prod_{i=1}^{N} \tilde{C}\left(u_{i}\right),
$$

where $\tilde{C}\left(u_{i}\right)=u^{-(M-1)} C(u)$, and $\langle\Omega| B(u)=0$.

From definitions (6) and (8) one finds by a direct calculation that $u^{M-1} B(u)$ and $u^{M-1} C(u)$ are polynomials in $u^{2}$ of degree $M-1$ :

$$
\begin{aligned}
& u^{M-1} B(u)=u^{2(M-1)} \mathcal{P}_{R}+\cdots+(-1)^{M-1} \sigma_{M}^{-} s_{M-1} \cdots s_{1}, \\
& u^{M-1} C(u)=u^{2(M-1)} \mathcal{P}_{L}+\cdots+(-1)^{M-1} s_{M} \cdots s_{2} \sigma_{1}^{+} .
\end{aligned}
$$

This decomposition allows us to express the steady states (4) and (5) through the state vectors (14) and (16), namely

$$
\left|S_{N}\right\rangle=\lim _{\{u\} \rightarrow \infty} \prod_{i=1}^{N} \tilde{B}\left(u_{i}\right)|\Omega\rangle, \quad\left\langle S_{N}\right|=\lim _{\{u\} \rightarrow \infty}\langle\Omega| \prod_{i=1}^{N} \tilde{C}\left(u_{i}\right) .
$$

The scalar product of the state vectors (14) and (16) may be evaluated directly by means of the commutation relations (10) with the help of Laplace formula for the determinant of the sum of two matrices. It also may be obtained as a special limit of the general formula for the scalar products (IX.6.26) of [3]. It should be mentioned that for the $R$-matrix (7) the auxiliary quantum fields (IX.6.24) commute and the answer for the scalar product has the determinantal form. It means that the scalar products of the state vectors of the integrable models associated with the $R$-matrix (7) are expressed as the determinants of the matrices with the entries which depend on $f$ and $g$ elements of $R$-matrix and on the eigenvalues $\alpha$ and $\delta(15)$ that characterize the particular model.

For the arbitrary variables $u, v \in \mathbb{C}$ the scalar product of the state vectors of the model is given by the following expression

$$
\begin{aligned}
& \left\langle\Psi\left(v_{1}, v_{2}, \ldots, v_{N}\right) \mid \Psi\left(u_{1}, u_{2}, \ldots, u_{N}\right)\right\rangle \\
& \quad=\left\{\prod_{j=1}^{N} \frac{1}{\left(v_{j} u_{j}\right)^{M-1}} \prod_{N \geq j>k \geq 1} \frac{v_{j} v_{k}}{v_{k}^{2}-v_{j}^{2}} \prod_{N \geq l>n \geq 1} \frac{u_{l} u_{n}}{u_{l}^{2}-u_{n}^{2}}\right\} \operatorname{det} Q .
\end{aligned}
$$


The entries of the $N \times N$ matrix $Q$ are

$$
Q_{j k}=\left\{v_{j}^{M}\left(u_{k}-u_{k}^{-1}\right)^{M}\left(\frac{u_{k}}{v_{j}}\right)^{N-1}-u_{k}^{M}\left(v_{j}-v_{j}^{-1}\right)^{M}\left(\frac{u_{k}}{v_{j}}\right)^{-N+1}\right\} \frac{1}{\frac{u_{k}}{v_{j}}-\left(\frac{u_{k}}{v_{j}}\right)^{-1}} .
$$

Using this representation and equalities (17) that represent the steady states through the state vectors of the model we can calculate the projection of the state vectors (14) and (16) on the steady states. For the left steady state we have

$$
\left\langle S_{N} \mid \Psi\left(u_{1}, u_{2}, \ldots, u_{N}\right)\right\rangle=\lim _{\{v\} \rightarrow \infty} \prod_{i=1}^{N}\left\langle\Psi\left(v_{1}, v_{2}, \ldots, v_{N}\right) \mid \Psi\left(u_{1}, u_{2}, \ldots, u_{N}\right)\right\rangle .
$$

This limit may be calculated with a help of the formula

$$
\lim _{v_{j} \rightarrow v} \frac{\operatorname{det}\left\{\Phi\left(v_{j}, u_{k}\right)\right\}}{\Delta(v)}=\operatorname{det}\left\{\frac{1}{j !} \frac{\partial^{j}}{\partial v^{j}} \Phi\left(v, u_{k}\right)\right\},
$$

where $\Phi(v, u)$ is an arbitrary differentiable for at least $N$ times function of two variables, and $\Delta(v)$ is the Vandermonde determinant. Taking the limit in (19) we obtain [31]:

$$
\left\langle S_{N} \mid \Psi\left(u_{1}, u_{2}, \ldots, u_{N}\right)\right\rangle=\prod_{k=1}^{N} u_{k}^{2} \prod_{N \geq l>n \geq 1} \frac{1}{u_{l}^{2}-u_{n}^{2}} \operatorname{det} V^{(M)},
$$

where $V^{(M)}$ is a $N \times N$ matrix with the entries equal to

$$
\begin{aligned}
V_{j k}^{(M)} & =\sum_{n=0}^{j-1}(-1)^{n}\left(\begin{array}{c}
M \\
n
\end{array}\right) u_{k}^{2(j-1-n)}, \quad 1 \leq j \leq N-1, \\
V_{N k}^{(M)} & =-\sum_{n=N-1}^{M}(-1)^{n}\left(\begin{array}{c}
M \\
n
\end{array}\right) u_{k}^{-2(n-N+1)} .
\end{aligned}
$$

The projection $\left\langle\Psi\left(u_{1}, u_{2}, \ldots, u_{N}\right) \mid S_{N}\right\rangle$ is given by the similar expression

$$
\begin{aligned}
& \left\langle\Psi\left(u_{1}, u_{2}, \ldots, u_{N}\right) \mid S_{N}\right\rangle=\prod_{k=1}^{N} u_{k}^{2} \prod_{N \geq n>l \geq 1} \frac{1}{u_{l}^{2}-u_{n}^{2}} \operatorname{det} \tilde{V}^{(M)}, \\
& \tilde{V}_{j k}^{(M)}=\sum_{n=0}^{N-j}(-1)^{n}\left(\begin{array}{c}
M \\
n
\end{array}\right) u_{k}^{2(N-j-n)}, \quad 2 \leq j \leq N, \\
& \tilde{V}_{1 k}^{(M)}=-\sum_{n=N}^{M}(-1)^{n}\left(\begin{array}{c}
M \\
n
\end{array}\right) u_{k}^{-2(n-N+1)} .
\end{aligned}
$$

\section{Form-factors}

The calculation of the correlation function (2) we start with the calculation of the matrix elements $\left\langle S_{N}\left|s_{1}\right| \Psi\left(u_{1}, u_{2}, \ldots, u_{N}\right)\right\rangle$ and $\left\langle\Psi\left(v_{1}, v_{2}, \ldots, v_{N}\right)\left|s_{1}\right| S_{N}\right\rangle$ which by means of $(17)$ are expressed through the form-factor of the projection operator, namely

$$
\begin{aligned}
& \left\langle S_{N}\left|s_{1}\right| \Psi\left(u_{1}, u_{2}, \ldots, u_{N}\right)\right\rangle=\lim _{\{v\} \rightarrow \infty}\left\langle\Psi\left(v_{1}, v_{2}, \ldots, v_{N}\right)\left|s_{1}\right| \Psi\left(u_{1}, u_{2}, \ldots, u_{N}\right)\right\rangle, \\
& \left\langle\Psi\left(v_{1}, v_{2}, \ldots, v_{N}\right)\left|s_{1}\right| S_{N}\right\rangle=\lim _{\{u\} \rightarrow \infty}\left\langle\Psi\left(v_{1}, v_{2}, \ldots, v_{N}\right)\left|s_{1}\right| \Psi\left(u_{1}, u_{2}, \ldots, u_{N}\right)\right\rangle .
\end{aligned}
$$


By the definition (8) the monodromy matrix may be expressed as

$$
T(u)=\left(\begin{array}{cc}
A_{M-1}(u) & B_{M-1}(u) \\
C_{M-1}(u) & D_{M-1}(u)
\end{array}\right)\left(\begin{array}{cc}
u s_{1} & \sigma_{1}^{-} \\
\sigma_{1}^{+} & u I-u^{-1} s_{1}
\end{array}\right) .
$$

From this formula it follows that the entries of the monodromy matrix may be expressed as

$$
\begin{aligned}
& A(u)=u A_{M-1}(u) s_{1}+B_{M-1}(u) \sigma_{1}^{+}, \\
& B(u)=A_{M-1}(u) \sigma_{1}^{-}+u B_{M-1}(u)-u^{-1} B_{M-1}(u) s_{1}, \\
& C(u)=u C_{M-1}(u) s_{1}+D_{M-1}(u) \sigma_{1}^{+}, \\
& D(u)=C_{M-1}(u) \sigma_{1}^{-}+u D_{M-1}(u)-u^{-1} D_{M-1}(u) s_{1} .
\end{aligned}
$$

Making use of the equalities $s_{j}^{2}=s_{j}, s_{j} \sigma_{j}^{-}=\sigma_{j}^{+} s_{j}=0$ we obtain, in particular, that

$$
s_{1} B(u)=\left(u-u^{-1}\right) B_{M-1}(u) s_{1}, \quad C(u) s_{1}=u s_{1} C_{M-1}(u) .
$$

Substituting the explicit form of the state vectors (14) and (16) into the form-factor (21) and taking into account the commutation relations (22) we find that for the arbitrary values of parameters $u, v$ the form-factor is proportional to the scalar product (18) of the state vectors on a lattice with $M-1$ sites:

$$
\begin{aligned}
& \left\langle\Psi\left(v_{1}, v_{2}, \ldots, v_{N}\right)\left|s_{1}\right| \Psi\left(u_{1}, u_{2}, \ldots, u_{N}\right)\right\rangle \\
& =\left\langle\Omega\left|\prod_{j=1}^{N} \tilde{C}\left(v_{j}\right) s_{1} \prod_{i=1}^{N} \tilde{B}\left(u_{i}\right)\right| \Omega\right\rangle=\left\langle\Omega\left|\prod_{j=1}^{N} \tilde{C}\left(v_{j}\right) s_{1}^{2} \prod_{i=1}^{N} \tilde{B}\left(u_{i}\right)\right| \Omega\right\rangle \\
& =\prod_{k=1}^{N}\left(1-u_{k}^{-2}\right)\left\langle\Omega\left|\prod_{j=1}^{N} \tilde{C}_{M-1}\left(v_{j}\right) \prod_{i=1}^{N} \tilde{B}_{M-1}\left(u_{i}\right)\right| \Omega\right\rangle .
\end{aligned}
$$

Taking the limit $\{v\} \rightarrow \infty$ we obtain

$$
\begin{aligned}
& \left\langle S_{L}\left|s_{1}\right| \Psi\left(u_{1}, u_{2}, \ldots, u_{N}\right)\right\rangle=\lim _{\{v\} \rightarrow \infty} \prod_{i=1}^{N}\left\langle\Psi\left(v_{1}, v_{2}, \ldots, v_{N}\right)\left|s_{1}\right| \Psi\left(u_{1}, u_{2}, \ldots, u_{N}\right)\right\rangle \\
& =\prod_{k=1}^{N}\left(1-u_{k}^{-2}\right) \lim _{\{v\} \rightarrow \infty}\left\langle\Omega\left|\prod_{j=1}^{N} \tilde{C}_{M-1}\left(v_{j}\right) \prod_{i=1}^{N} \tilde{B}_{M-1}\left(u_{i}\right)\right| \Omega\right\rangle \\
& =\prod_{k=1}^{N}\left(u_{k}^{2}-1\right) \prod_{N \geq l>n \geq 1} \frac{1}{u_{l}^{2}-u_{n}^{2}} \operatorname{det} V^{(M-1)},
\end{aligned}
$$

where the entries of $N \times N$ matrix $V^{(M-1)}$ are (20) with $M$ replaced on $M-1$. Respectively we have

$$
\left\langle\Psi\left(u_{1}, u_{2}, \ldots, u_{N}\right)\left|s_{1}\right| S_{N}\right\rangle=\prod_{k=1}^{N} u_{k}^{2} \prod_{N \geq n>l \geq 1} \frac{1}{u_{l}^{2}-u_{n}^{2}} \operatorname{det} \tilde{V}^{(M-1)} .
$$

\section{$5 \quad$ Bethe ansatz solution}

By following through the arguments of the algebraic Bethe ansatz method $[1,2,3]$ one proves that the state vectors (14) and (16) are the right and the left eigenstates of the transfer matrix (11) with the same eigenvalues

$$
\tau(v)\left|\Psi\left(u_{1}, u_{2}, \ldots, u_{N}\right)\right\rangle=\Theta_{N}(v,\{u\})\left|\Psi\left(u_{1}, u_{2}, \ldots, u_{N}\right)\right\rangle,
$$




$$
\left\langle\Psi\left(u_{1}, u_{2}, \ldots, u_{N}\right)\right| \tau(v)=\left\langle\Psi\left(u_{1}, u_{2}, \ldots, u_{N}\right)\right| \Theta_{N}(v,\{u\})
$$

if allowed parameters $u_{1}, u_{2}, \ldots, u_{N}$ in $(25)$ satisfy Bethe equations $[14,15,16,17,18,19]$ :

$$
\left(1-u_{n}^{-2}\right)^{-M} u_{n}^{-2 N}=(-1)^{N-1} \prod_{j=1}^{N} u_{j}^{-2} \equiv(-1)^{N-1} U^{-2} .
$$

The eigenvalues $\Theta_{N}(v,\{u\})$ are equal to

$$
\Theta_{N}(v,\{u\})=\prod_{j=1}^{N} \frac{u_{j}^{2}}{u_{j}^{2}-v^{2}}+\left(1-v^{-2}\right)^{M} \prod_{j=1}^{N} \frac{v^{2}}{v^{2}-u_{j}^{2}} .
$$

From the definition of the cyclic shift operator (12) it follows that its eigenvalues are equal to

$$
\Theta_{N}(1,\{u\})=\prod_{j=1}^{N} \frac{1}{1-u_{j}^{-2}}
$$

From (13) one finds the eigenenergies of the Hamiltonian (1)

$$
E_{N}=-\left.\frac{1}{2} \Theta_{N}^{-1}(1,\{u\}) \frac{\partial}{\partial v} \Theta_{N}(v,\{u\})\right|_{v=1}=-\sum_{j=1}^{N} \frac{1}{u_{j}^{2}-1} .
$$

The obvious solution $u_{1}=u_{2}=\cdots=u_{N}=\infty$ provides the stationary solution with the eigenvalue $E_{N}=0$. The steady state $\left|S_{N}\right\rangle$ is the eigenvector of the transfer matrix (11) $\tau(u)$ with the eigenvalue equal to one:

$$
\tau(u)\left|S_{N}\right\rangle=\Theta_{N}(v,\{\infty\})\left|S_{N}\right\rangle=\left|S_{N}\right\rangle
$$

and hence it is stochastic.

The scalar product of eigenvectors (14), (16) is found from formula (18) with $v_{j}=u_{j}$ satisfying Bethe equations (26). Understanding the diagonal elements of the matrix $Q$ in the sense of L'Hôpitall rule one obtains the following expression for the norm of any eigenvector:

$$
\mathcal{N}^{2}\left(u_{1}, u_{2}, \ldots, u_{N}\right)=\left\langle\Psi\left(u_{1}, u_{2}, \ldots, u_{N}\right) \mid \Psi\left(u_{1}, u_{2}, \ldots, u_{N}\right)\right\rangle=U^{2 N} \prod_{l \neq n} \frac{1}{u_{l}^{2}-u_{n}^{2}} \operatorname{det} \tilde{Q}
$$

with the entries of the matrix $\tilde{Q}$ equal to

$$
\tilde{Q}_{j k}=\frac{N-1+(M-N+1) u_{j}^{-2}}{1-u_{j}^{-2}} \delta_{j k}-\left(1-\delta_{j k}\right) .
$$

For the special solution $u_{1}=u_{2}=\cdots=u_{N}=\infty$ the norm of eigenvectors is equal to $Z_{N}$.

There is $Z_{N}$ solutions of the Bethe equations [16], and the state vectors belonging to the different sets of the solutions of the Bethe equations are orthogonal. The eigenvectors (25) provide the resolution of the identity operator

$$
I=\sum_{\{u\}} \frac{\left|\Psi\left(u_{1}, u_{2}, \ldots, u_{N}\right)\right\rangle\left\langle\Psi\left(u_{1}, u_{2}, \ldots, u_{N}\right)\right|}{\mathcal{N}^{2}\left(u_{1}, u_{2}, \ldots, u_{N}\right)},
$$

where the summation is over all different solutions of Bethe equations (26). 


\section{Stationary correlation function}

For the transitionally invariant system the form-factor of the projection operator $s_{m}$ is expressed through the form-factor of the operator $s_{1}$ :

$$
\begin{aligned}
\left\langle\Psi\left(u_{1}, u_{2}, \ldots, u_{N}\right)\left|s_{m}\right| S_{N}\right\rangle & =\left\langle\Psi\left(u_{1}, u_{2}, \ldots, u_{N}\right)\left|\tau^{m-1} s_{1} \tau^{1-m}\right| S_{N}\right\rangle \\
& =\prod_{j=1}^{N}\left(1-u_{j}^{-2}\right)^{1-m}\left\langle\Psi\left(u_{1}, u_{2}, \ldots, u_{N}\right)\left|s_{1}\right| S_{N}\right\rangle,
\end{aligned}
$$

where the property (27) was used.

The substitution of the resolution of the identity into $\left\langle S_{N}\left|s_{1} e^{-|t| H} s_{m}\right| S_{N}\right\rangle$ gives

$$
\begin{aligned}
\left\langle S_{N}\left|s_{1} e^{-|t| H} s_{m}\right| S_{N}\right\rangle & =\sum_{\{u\}} \frac{\left\langle S_{N}\left|s_{1} e^{-|t| H}\right| \Psi\left(u_{1}, u_{2}, \ldots, u_{N}\right)\right\rangle\left\langle\Psi\left(u_{1}, u_{2}, \ldots, u_{N}\right)\left|s_{m}\right| S_{N}\right\rangle}{\mathcal{N}^{2}\left(u_{1}, u_{2}, \ldots, u_{N}\right)} \\
& =\sum_{\{u\}} e^{-|t| E_{N}} \frac{\left\langle S_{N}\left|s_{1}\right| \Psi\left(u_{1}, u_{2}, \ldots, u_{N}\right)\right\rangle\left\langle\Psi\left(u_{1}, u_{2}, \ldots, u_{N}\right)\left|s_{1}\right| S_{N}\right\rangle}{\mathcal{N}^{2}\left(u_{1}, u_{2}, \ldots, u_{N}\right) \prod_{j=1}^{N}\left(1-u_{j}^{-2}\right)^{m-1}} .
\end{aligned}
$$

From the determinantal representations of the matrix elements (23), (24) and of the norm (28) we finally obtain the answer for the stationary correlation function (2):

$$
\begin{aligned}
\frac{1}{Z_{N}}\left\langle S_{N}\left|s_{1} e^{-|t| H} s_{m}\right| S_{N}\right\rangle= & \left(\frac{M-N}{M}\right)^{2} \\
& +\sum_{\{u\}} \frac{e^{-|t| E_{N}}}{U^{2 N}} \prod_{j=1}^{N} \frac{\left(u_{j}^{2}-1\right) u_{j}^{2}}{\left(1-u_{j}^{-2}\right)^{m-1}} \frac{\operatorname{det} \tilde{V}^{(M-1)} \operatorname{det} V^{(M-1)}}{\operatorname{det} \tilde{Q}},
\end{aligned}
$$

where the summation is taken over all different solutions of Bethe equations (26) except the special one. The first term on the r.h.s. is the contribution of the stationary state.

\section{Conclusive remarks}

The models connected with the 'crystal base' $R$-matrix (7) naturally appear in the theory of the boxed plane partitions - three-dimensional Young diagrams placed into a box of a finite size, and in the theory of random walkers $[32,8]$. Some aspects of these connections were discussed in the papers [33, 34, 35, 36, 37, 38].

In its turn plane partitions and random walkers are employed in analyzes of the models of statistical physics describing faceted crystals [39], direct percolation [40], one-dimensional growth processes [41]. It emphasizes the importance of the mentioned integrable models in the theory of the non-equilibrium processes.

\section{Acknowledgements}

The work was partially supported by the RFBR grant 07-01-00358. 


\section{References}

[1] Faddeev L.D., Quantum completely integrable models in field theory, Soviet Sci. Rev. Sect. C: Math. Phys. Rev., Vol. 1, Harwood Academic, Chur, 1980, 107-155.

[2] Kulish P.P., Sklyanin E.K., Quantum spectral transform method. Recent developments, Lecture Notes in Phys., Vol. 151, Springer, Berlin - New York, 1982, 61-119.

[3] Korepin V.E., Bogoliubov N.M., Izergin A.G., Quantum inverse scattering method and correlation functions, Cambridge Monographs on Mathematical Physics, Cambridge University Press, Cambridge, 1993.

[4] Drinfel'd V.G., Quantum groups, in Proceedings of the International Congress of Mathematicians, Vols. 1, 2 (Berkeley, Calif., 1986), Amer. Math. Soc., Providence, RI, 1987, 798-820.

[5] Faddeev L.D., Reshetikhin N.Yu., Takhtajan L.A., Quantisation of Lie groups and Lie algebras, Leningrad Math. J. 1 (1990), 193-225.

[6] Kashivara M., Crystalizing the q-analogue of universal enveloping algebras, Comm. Math. Phys. 133 (1990), 249-260.

[7] Stanley R., Enumerative combinatorics, Vol. 2, Cambridge Studies in Advanced Mathematics, Vol. 62, Cambridge University Press, Cambridge, 1999.

[8] Bressoud D.M., Proofs and confirmations. The story of the alternating sign matrix conjecture, Cambridge University Press, Cambridge, 1999.

[9] Evans M.R., Blythe R.A., Nonequilibrium dynamics in low-dimensional systems, Phys. A 313 (2002), 110152, cond-mat/0110630.

[10] Schütz G., Exactly solvable models for many-body systems far from equilibrium, Phase Transitions and Critical Phenomena, Vol. 19, Editors C. Domb and J.L. Lebowitz, Academic Press, San Diego, CA, 2001, $1-251$.

[11] Korepin V., Dual field formulation of quantum integrable models, Comm. Math. Phys. 113 (1987), 177-190.

[12] Bogoliubov N.M., Izergin A.G., Kitanine N.A., Correlators of the phase model, Phys. Lett. A 231 (1997), 347-352, solv-int/9612002.

[13] Bogoliubov N.M., Izergin A.G., Kitanine N.A., Correlation functions for a strongly correlated boson system, Nuclear Phys. B 516 (1998), 501-528, solv-int/9710002.

[14] Gwa L.-H., Spohn H., Bethe solution for the dynamical-scaling exponent of the noisy Burgers equation, Phys. Rev. A 46 (1992), 844-854.

[15] Derrida B., Lebowitz J., Exact large deviation function in the asymmetric exclusion process, Phys. Rev. Lett. 80 (1998), 209-213, cond-mat/9809044.

[16] Golinelli O., Mallick K., Bethe ansatz calculation of the spectral gap of the asymmetric exclusion process, J. Phys. A: Math. Gen. 37 (2004), 3321-3331, cond-mat/0312371.

[17] Golinelli O., Mallick K., Spectral gap of the totally asymmetric exclusion process at arbitrary filling, J. Phys. A: Math. Gen. 38 (2005), 1419-1425, cond-mat/0411505.

[18] de Gier J., Essler F.H.L., Bethe ansatz solution of the asymmetric exclusion process with open boundaries, Phys. Rev. Lett. 95 (2005), 240601, 4 pages, cond-mat/0508707.

[19] Prolhac S., Mallick K., Current fluctuations in the exclusion process and Bethe ansatz, J. Phys. A: Math. Theor. 41 (2008), 175002, 20 pages, arXiv:0801.4659.

[20] Noh J.D., Kim D., Interacting domain walls and the five-vertex model, Phys. Rev. E 49 (1995), 1943-1961, cond-mat/9312001.

[21] Kim D., Bethe Ansatz solution for crossover scaling functions of the asymmetric $X X Z$ chain and the Kardar-Parisi-Zhang-type growth model, Phys. Rev. E 52 (1995), 3512-3524, cond-mat/9503169.

[22] Lee D.S., Kim D., Large deviation function of the partially asymmetric exclusion process, Phys. Rev. E 59 (1999), 6476-6482, cond-mat/9902001.

[23] Bogoliubov N.M., Nassar T., On the spectrum of the non-Hermitian phase-difference model, Phys. Lett. A 234 (1997), 345-350.

[24] Golinelli O., Mallick K., The asymmetric simple exclusion process: an integrable model for non-equilibrium statistical mechanics, J. Phys. A: Math. Gen. 39 (2006), 12679-12705, cond-mat/0611701.

[25] Schütz G., Exact solution of the master equation for the asymmetric exclusion process, J. Statist. Phys. $8 \mathbf{8}$ (1997), 427-445. 
[26] Borodin A., Ferrari P., Prahofer M., Sasamoto T., Fluctuation properties of the TASEP with periodic initial configuration, J. Statist. Phys. 129 (2007), 1055-1080, math-ph/0608056.

[27] Sasamoto T., Spatial correlations of the 1D KPZ surface on a flat substrate, J. Phys. A: Math. Gen. 38 (2005), L549-L556, cond-mat/0504417.

[28] Prähofer M., Spohn H., Current fluctuations for the totally asymmetric simple exclusion process, in In and Out of Equilibrium (Mambucaba, 2000), Editor V. Sidoravicius, Progr. Probab., Vol. 51, Birkhäuser Boston, Boston, MA, 2002, 185-204, cond-mat/0101200.

[29] Prähofer M., Spohn H., Exact scaling functions for one-dimensional stationary KPZ growth, J. Statist. Phys. 115 (2004), 255-279.

[30] Schütz G., Duality relations for asymmetric exclusion processes, J. Statist. Phys. 86 (1997), 1265-1287.

[31] Bogoliubov N.M., Five vertex model with the fixed boundary conditions, Algebra i Analiz 21 (2009), 58-78.

[32] Macdonald I.G., Symmetric functions and Hall polynomials, Oxford Mathematical Monographs. Oxford Science Publications, The Clarendon Press, Oxford University Press, New York, 1995.

[33] Bogoliubov N.M., Boxed plane partitions as an exactly solvable boson model, J. Phys. A: Math. Gen. 38 (2005), 9415-9430, cond-mat/0503748.

[34] Bogoliubov N.M., Enumeration of plane partitions and the algebraic Bethe ansatz, Theoret. and Math. Phys. 150 (2007), 165-174.

[35] Bogoliubov N.M., Four-vertex model and random tilings, Theoret. and Math. Phys. 155 (2008), 523-535, arXiv:0711.0030.

[36] Tsilevich N., Quantum inverse method for the $q$-boson model, and symmetric functions, Funct. Anal. Appl. 40 (2006), 207-217, math-ph/0510073.

[37] Shigechi K., Uchiyama M., Boxed skew plane partition and integrable phase model, J. Phys. A: Math. Gen. 38 (2005), 10287-10306, cond-mat/0508090.

[38] Bogoliubov N.M., Integrable models for vicious and friendly walkers, J. Math. Sci. (N.Y.) 143 (2007), 2729-2737.

[39] Ferrari P.L., Spohn H., Step functions for a faceted crystal, J. Statist. Phys. 113 (2003), 1-46.

[40] Rajesh R., Dhar D., An exactly solvable anisotropic directed percolation model in three dimensions, Phys. Rev. Lett. 81 (1998), 1646-1649, cond-mat/9808023.

[41] Kardar M., Parisi G., Zhang Y.Z., Dynamic scaling of growing interfaces, Phys. Rev. Lett. 56 (1986), 889-892. 